Journal of Antimicrobial Chemotherapy (1993) 31, Suppl. E, 81-88

\title{
Multicentre evaluation of azithromycin in comparison with co-amoxiclav for the treatment of acute otitis media in children
}

\author{
U. B. Schaad \\ Division of Infectious Disease, Department of Paediatrics, University of Bern, \\ Inselspital, CH-3010 Bern, Switzerland
}

\begin{abstract}
A total of 389 children (age 0-6-10-2 years) with typical signs and symptoms of acute otitis media were randomized $(1: 1)$ to treatment with either azithromycin or coamoxiclav. The dosage schedule for azithromycin was $10 \mathrm{mg} / \mathrm{kg} /$ day, in a single daily dose, administered for three days. Co-amoxiclav was given at a dose of $13.3 \mathrm{mg} / \mathrm{kg}$ (amoxycillin equivalent) tid for ten days. Patients were evaluated 4-6 days and 12-16 days after the start of therapy. A satisfactory clinical response was reported for $93.2 \%$ of the 192 evaluable azithromycin-treated patients (144 cured, 35 improved), and for $97.3 \%$ of the 189 evaluable co-amoxiclav-treated patients (148 cured, 36 improved). Six (3.0\%) relapses occurred in the azithromycin group, and four $(2.1 \%)$ in the co-amoxiclav treatment group, respectively. Side-effects were recorded in a significantly fewer number of the azithromycin patients $(23$ of $197 ; 11.7 \%)$ compared with the co-amoxiclav patients (43 of 192; $22.4 \%, P<0-02$ ). Adverse events were mainly gastrointestinal in nature, with diarrhoea the most frequent complaint $(32$ cases with co-amoxiclav; five with azithromycin; $P<0-001$ ). One patient from each group discontinued therapy because of treatment-related adverse events. Laboratory analyses (mainly haematological in nature) showed abnormalities in six of 100 azithromycin patients and ten of 101 co-amoxiclav patients. It was concluded that three-day, single-dose azithromycin and ten-day tid co-amoxiclav therapy have comparable clinical efficacy in paediatric patients with acute otitis media; however, there was a lower incidence of side effects in the azithromycin group.
\end{abstract}

\section{Introduction}

Azithromycin is a new once-daily antibiotic which is chemically related to erythromycin. The use of erythromycin in the treatment of upper respiratory tract infections (URTI) has remained an area of controversy because of its poor activity against Haemophilus influenzae (Washington \& Wilson, 1985). However, azithromycin is at least four-fold more active than erythromycin against this pathogen, whilst retaining a similar overall spectrum of activity against common Gram-positive organisms and atypical respiratory pathogens (Table I). The clinical benefits of once-daily dosing for five days with azithromycin ( $500 \mathrm{mg}$ on day 1 , followed by $250 \mathrm{mg}$ /day on days $2-5$ ) for URTI in adults have been demonstrated (Casiano, 1991; Felstead et al., 1991; Hooton, 1991). Felstead et al. (1991) concluded, from the results of two multicentre studies, that azithromycin tissue levels were sufficient to eradicate $H$. influenzae, Streptococcus pneumoniae and Staphylococcus aureus in URTI. In children with otitis media, five-day azithromycin therapy $(10 \mathrm{mg} / \mathrm{kg}$ on day $1 ; 5 \mathrm{mg} / \mathrm{kg} /$ day on days $2-5)$ was significantly more effective than a ten-day regimen of co-amoxiclav (Daniel et al., 1990). Further

81 
Table I. The in-vitro spectrum of azithromycin and erythromycin against common respiratory pathogens.

\begin{tabular}{lcc}
\hline & \multicolumn{2}{c}{$\mathrm{MIC}_{\text {od }}(\mathrm{mg} / \mathrm{L})$} \\
\cline { 2 - 3 } Organism & azithromycin & erythromycin \\
\hline Streptococcus pyogenes & $0-05$ & $0-03$ \\
S. pneumoniae & $0-05$ & $0-03$ \\
S. aureus & 1.0 & $0-25$ \\
H. influenzae & $0-5$ & 4.00 \\
Moraxella catarrhalis & $0-03$ & $0-25$ \\
\hline
\end{tabular}

Data from: Retsema el al. (1987); Rylander \& Hallander (1988); Maskell, Sefton \& William (1990); Edelstein \& Edelstein (1991); Felstead et al. (1991).

trials are now being conducted to confirm these findings in URTI with a three-day azithromycin regimen.

The aim of this multicentre study was to evaluate the efficacy and safety of a threeday course of azithromycin in comparison with a standard ten-day course of coamoxiclav for the treatment of children with acute otitis media.

\footnotetext{
Methods

Study enrolment

Children, 2-12 years of age, suffering from acute otitis media were eligible for entry into the study. The study protocol was reviewed and approved by the Medical Ethics Committee of the Department of Paediatrics, University of Bern. After obtaining informed consent from parents or legal guardians, the medical history of each child was recorded and a full physical examination carried-out at the pre-treatment clinical visit (visit 1). Diagnosis of otitis media was based on the presence of at least two of the following symptoms: earache; reduced general condition or headache or excitation; fever; ear noise or conductive hearing impairment. Patients also had to exhibit at least one of the following clinical signs: radial injection or diffuse redness of the eardrum; bulging of the tympanic membrane; rupture of the tympanic membrane; or otopyorrhoea. Signs and symptoms were graded according to the following severity scale: 0 (not present), 1 (mild), 2 (moderate), and 3 (severe). Microbiological examinations were not made since Swiss paediatricians rarely obtain material by myringotomy when diagnosing and treating acute otitis media.

Laboratory tests were carried out if medically indicated or accepted by the parents or guardians. These tests included a complete blood count, including differential leucocyte and platelet counts, as well as serum enzymes (SGOT, SGPT and alkaline phosphatase), blood urea nitrogen, and bilirubin.

Treatment with another investigational drug within four weeks, or other antibiotics within two weeks, before study enrolment (unless there was documented failure with treatment), was a basis for exclusion from the study. Patients were also excluded if they had a known hypersensitivity to macrolides, azithromycin or $\beta$-lactam antibiotics, or if
} 
they showed evidence of any gastrointestinal condition that could affect absorption of the study drugs.

\section{Study drugs}

Patients who satisfied the study criteria were assigned randomly $(1: 1)$ to receive oral therapy with either azithromycin or co-amoxiclav. Patients assigned to azithromycin received treatment for three consecutive days: $10 \mathrm{mg} / \mathrm{kg} /$ day in one daily dose, administered as an oral suspension $1 \mathrm{~h}$ before or $2 \mathrm{~h}$ after a meal. The daily dose of coamoxiclav was $40 \mathrm{mg} / \mathrm{kg}$ body weight (amoxycillin equivalent), administered as an oral suspension in three divided doses for ten days. Treatment started no more than $24 \mathrm{~h}$ following the first clinical assessment (visit 1).

\section{Efficacy and safety assessments}

Clinical evaluations were carried out at baseline, at days 4-6 (visit 2), and 12-16 (visit 3 ) after the start of therapy. Patients were included in the efficacy evaluation if they satisfied all entry criteria, received sufficient exposure to drug therapy (three days for azithromycin and at least seven days for co-amoxiclav) and were re-evaluated at a final follow-up visit, 7-20 days after the start of therapy.

Efficacy was assessed on the basis of the severity of key signs (reddened eardrum, diminished light reflex and perforated eardrum) and symptoms (earache, lethargy or malaise, irritability) at each visit. Clinical efficacy at the end of therapy (day 7-20) was graded as cure, improvement, failure or relapse. 'Cure' was the disappearance of baseline symptoms of infection; 'improvement' a partial disappearance of symptoms; and 'failure' no change in, or worsening of, symptoms from baseline. 'Relapse' was recorded when there was an improvement or disappearance of pretreatment signs and symptoms, followed by their worsening or reappearance.

The evaluation of safety and tolerance was based on a record of adverse events and laboratory test abnormalities during the study period (up to 20 days after the start of therapy). At each visit, all adverse events either reported by the patient or parent or guardian, or observed by the investigator, were recorded with information on their onset date, duration, severity, outcome, and relation to the study drug. To avoid eliciting specific complaints or side-effects, the patient's parent or guardian was asked only general questions. Laboratory test abnormalities were reviewed after the start of therapy and at the last visit.

\section{Statistical analyses}

Data from all participating centres were pooled for safety and efficacy analyses. All statistical tests were two-tailed. Baseline characteristics of the two treatment groups were compared by a Student's t-test or Chi-squared test, as appropriate. The distribution of clinical response (cured, improved, failed) for the two treatment groups was compared by Cochran-Mantel-Haenszel statistics based on Ridit scores (Bross, 1958), with $95 \%$ confidence intervals for clinical success rates (cure plus improvement). Between group differences were considered to be significant if $P<0.05$. 
Table II. Baseline demographic data for 389 patients.

\begin{tabular}{lcc}
\hline & Azithromycin & Co-amoxiclav \\
\hline Number of patients & 197 & 192 \\
Males/females & $110 / 87$ & $121 / 71$ \\
Mean age (years) & 4.5 & $4 \cdot 4$ \\
Age range & $0-6-10.2$ & $0-6-9.2$ \\
Age distribution & & \\
$<2$ years & $14(7 \cdot 1 \%)$ & $15(7.8 \%)$ \\
$2-5$ years & $105(53.3 \%)$ & $108(56.3 \%)$ \\
$5-11$ years & $78(39.6 \%)$ & $69(35.9 \%)$ \\
Race & & \\
Caucasian & 187 & 175 \\
Afro-Caribbean & 2 & 5 \\
Asian & 3 & 8 \\
Other & 5 & 4 \\
\hline
\end{tabular}

\section{Results}

Baseline characteristics and exclusions from evaluation

A total of 197 children with mean age 4.5 years (range $0.6-10.2$ years) and typical signs and symptoms of acute otitis media were assigned randomly to the azithromycin group, and 192 children with mean age 4.4 years (range 0.6-9.2 years) to the co-amoxiclav group (Table II).

Eight patients (five azithromycin; three co-amoxiclav) discontinued treatment early and were excluded from the efficacy analysis. Two children treated with azithromycin discontinued because of vomiting; this was considered to be drug-related in one case, but was attributed to viral gastritis in the other. The third child in the azithromycin group was withdrawn on request by the parents because fever was still high $12 \mathrm{~h}$ after the start of treatment, though this was not considered a treatment failure by the physician. One azithromycin patient was withdrawn because of treatment-related sideeffects. Two patients (one from each treatment group) were not included in the efficacy analysis because of non-compliance. Two other patients receiving co-amoxiclav treatment were withdrawn because of, respectively, a diagnosis of mumps and because the inclusion criteria were not met. An additional patient in the co-amoxiclav group interrupted treatment on day 8 because of diarrhoea, but was included in the efficacy analysis.

Concomitant medications (mostly non-steroidal anti-inflammatory drugs and paracetamol) were used during the study by 110 patients ( 54 azithromycin; $56 \mathrm{co}$ amoxiclav). None of these patients received systemic antibiotics other than the study drugs.

\section{Efficacy}

In total, 192 patients treated with azithromycin and 189 patients treated with coamoxiclav were eligible for efficacy evaluation at day 7-20 (Table III). A satisfactory clinical response (cure or improvement by the final visit) was recorded in $93.2 \%$ (179 of 192) azithromycin patients and $97.3 \%$ (185 of 189) co-amoxiclav patients. There was no statistically significant difference in cure, improvement, failure or relapse rates between 
Table III. Assessment of clinical response at the end of therapy (day 12-16) in clinically evaluable patients

\begin{tabular}{lcc}
\hline & \multicolumn{2}{c}{ No. of patients (\%) } \\
& azithromycin & co-amoxiclav \\
\hline Cure & $144(75 \cdot 0 \%)$ & $148(78 \cdot 3 \%)$ \\
Improvement & $35(18.2 \%)$ & $36(19.0 \%)$ \\
Relapse & $6(3 \cdot 1 \%)$ & $4(2 \cdot 1 \%)$ \\
Failure & $6(3 \cdot 1 \%)$ & $1(0.5 \%)$ \\
Undetermined & $1(0.5 \%)$ & 0 \\
Total evaluable & $192(100 \%)$ & $189(100 \%)$ \\
\hline
\end{tabular}

This patient had chronic serosal otitis media and was excluded from the statistical analysis.

the two treatment groups $(P=0-23)$. Six azithromycin and four co-amoxiclav patients relapsed 8-13 days and 4-7 days, respectively, after the end of therapy. One azithromycin patient with chronic serosal otitis media had an undetermined clinical response, and was excluded from the statistical analysis. There was one treatment failure in the co-amoxiclav group. This patient's (age 2.5 years) condition worsened during followup, and he received cefaclor treatment which produced a clinical cure. Six azithromycin patients were graded as treatment failures, with no improvement in the signs of otitis media compared with baseline assessments. Three of these treatment failures were recorded in one centre. Two patients were found to have perforated eardrums at the first follow-up evaluation. No follow-up data were available for three patients: two patients improved following cefaclor and co-amoxiclav treatment, respectively; and one patient treated with amoxycillin showed an improvement.

Of 14 children aged $<2$ years from each group who were evaluable for efficacy analysis, the distribution of response was the same (nine cured, three improved, two relapsed). There were no treatment failures recorded in this lower age group.

Thirty azithromycin patients and 31 co-amoxiclav patients presented with eardrum perforation at baseline. Perforation also occurred between baseline and the second visit in four additional azithromycin patients (this occurred on the first day of active treatment in three children). Children with this condition responded equally well to both agents: eardrum perforations decreased in size or were healed in 24 of 30 azithromycin-treated patients and in 26 of 31 co-amoxiclav-treated patients at the last follow-up visit. All patients with bilateral eardrum perforation responded to azithromycin treatment (two cured, one improved) and co-amoxiclav treatment (four cured, one improved).

\section{Safety and toleration}

The severity and incidence of treatment-related adverse events is summarized in Table IV. Overall, patients treated with azithromycin reported significantly fewer and less severe side-effects than those treated with co-amoxiclav $(P<0.02)$. Treatment-related side-effects were reported in $23(11.7 \%)$ of the 197 azithromycin-treated patients. These side-effects were mostly gastrointestinal (five diarrhoea, seven abdominal pain, and three vomiting), but there were five cases of cutaneous events and six cases of general 
Tabłe IV. Incidence and severity of treatment-related side-effects

\begin{tabular}{lcccc}
\hline & \multicolumn{2}{c}{$\begin{array}{c}\text { Azithromycin } \\
(n=197)\end{array}$} & $\begin{array}{c}\text { Co-amoxiclav } \\
(n=192)\end{array}$ \\
\cline { 2 - 5 } & $\begin{array}{c}\text { No. } \\
\text { of } \\
\text { patients }\end{array}$ & $\begin{array}{c}\text { No. } \\
\text { of } \\
\text { reports }\end{array}$ & $\begin{array}{c}\text { No. } \\
\text { of } \\
\text { patients }\end{array}$ & $\begin{array}{c}\text { No. } \\
\text { of } \\
\text { reports }\end{array}$ \\
\hline Side-effects & 5 & 5 & 7 & 8 \\
Psin or appendages & 0 & 0 & 1 & 1 \\
Gastrointestinal & 15 & 17 & 36 & 44 \\
General & 6 & 6 & 1 & 1 \\
Total patients & $23(11 \cdot 7 \%)$ & $43(22.4 \%)$ & \\
\hline
\end{tabular}

fatigue, nervousness and irritability. All azithromycin-related side-effects were mild, with the exception of one case of vomiting which led to withdrawal from the study.

In the co-amoxiclav group, $43(22.4 \%)$ of 192 patients reported side-effects. The incidence of diarrhoea was significantly more common in this treatment group (32 vs 5 patients; $P<0.001$ ). Other gastrointestinal side-effects included vomiting (five patients), abdominal pain (three patients), nausea (three patients) and anorexia (one patient). In addition, seven patients had adverse events relating to the skin, and fatigue was reported in one patient. The majority of side-effects were mild to moderate in nature, except four which were classed as severe (one vomiting, two diarrhoea and one urticaria); one patient with severe diarrhoea discontinued treatment. Seven of the 15 patients in this treatment group who were aged $<2$ years presented with side-effects (one exanthema, five diarrhoea, one vomiting).

Laboratory test results were available for 100 azithromycin-treated and 101 coamoxiclav-treated patients. One patient in the azithromycin group showed a clinically significant drop in neutrophil count, but the treating physician did not consider this change to be drug related. Six patients in the co-amoxiclav group had clinically significant laboratory abnormalities, and in two of these cases a drug-related effect could not be excluded.

\section{Discussion}

The results of this study in children with acute otitis media indicate that three-day azithromycin is clinically equivalent to ten-day treatment with co-amoxiclav, but that azithromycin is better tolerated. There was a significantly higher incidence of both gastrointestinal and adverse events relating to the skin in the co-amoxiclav treatment group. Bacteriological eradication rates were not recorded because myringotomy is not carried out routinely in Switzerland in cases of uncomplicated acute otitis media in children. In $>50 \%$ of cases, otitis media is caused by $S$. pneumoniae and $H$. influenzae; consequently, current practice recommends the use of tympanocentesis only if there is no improvement following therapy for 2-3 days, or in recurrent infections, or in patients with underlying disease states.

Azithromycin appears to be a promising new therapy for children. Acceptance, by both parents and their sick children, of the once-daily regimen and the three-day duration of azithromycin treatment was excellent. The use of antibiotics with a shorter, 
less frequent dosing to improve patient compliance should be an important consideration in this patient group (Greenberg, 1984; Cockbum et al., 1987). The relationship between compliance and duration of therapy was studied by Bergman \& Werner (1963) in children with otitis media. After treatment for the first 3-5 days, non-compliance was recorded in approximately $50 \%$ of patients, this rose significantly to $71 \%$ after treatment for 6-7 days (Bergman \& Werner, 1963). However, compliance also depends on the motivation of the mother, or other care-giver, and her perception of the severity of the child's illness (Gordis, Markowitz \& Lilienfeld, 1969; Becker, Drachman \& Kirscht, 1972).

In this study, one child was withdrawn from the study early because of anxiety by the mother. However, pharmacokinetic findings provide evidence that azithromycin continues to act at the site of infection for several days after the end of dosing (Foulds, Shepard \& Johnson, 1990). Thus, prolonged therapeutic benefit would be expected. Offering information to the parent or care-giver regarding therapy has been shown to be an important determinant in improving patient compliance in children (Lima et al., 1976; Finney et al., 1985). Three-day, three-dose azithromycin therapy, together with appropriate parental guidance, should therefore go a long way towards achieving optimum compliance and clinical outcome in children with acute otitis media.

\section{Acknowledgements}

The author wishes to acknowledge all members of the Azithromycin Study Group. The active participants in this Swiss multicentre trial were: Dr Claude Godard, Monthey; Dr Christine Bammatter, Epalinges; Dr Jean-Pierre Christen, Nyon; Dr Francois Besson, Fribourg; Dr Patrick Diebold, Monthey; Dr Serban Sichitiu, Lausanne; Dr Dimitris Sidiropoulos, Bern; Dr Vittorio Sautter, Bern; Dr Etienne Joss, Bern; Dr Meinrad Ryffel, Münchenbuchsee; Dr Rainer Kobelt, Wabern; Dr Hansjörg Schneider, Bern; Dr Andreas Zimmermann, Belp; Dr Rudolf Kōnig, Ostermundigen; Dr Heinz Brauer, Schönbühl; Dr Jacques Périsset, Estavayer-le-Lac; Dr Petrign Töndury, Bern; Dr Björn H. Straume, Bern; Dr Vincenzo D'Apuzzo, Mendrisio; Dr Maria Pia Gianinazzi, Lugano; Dr Domenico Giuseppe Fontana, Lamone; Dr Michele Raggi, Massagno.

\section{References}

Becker, M. H., Drachman, R. H. \& Kirscht, J. P. (1972). Predicting mothers' compliance with pediatric medical regimens. Pediatrics 81, 843-54.

Bergman, A. B. \& Werner, R. J. (1963). Failure of children to receive penicillin by mouth. New England Journal of Medicine 268, 1334-8.

Bross, I. D. J. (1958). How to use the Ridit analysis. Biometrics 14, 18-38.

Casiano, R. R. (1991). Azithromycin versus amoxicillin in the treatment of acute maxillary sinusitis. American Journal of Medicine 91, Suppl. 3A, 27S-30S.

Cockburn, J., Gibberd, R. W., Reid, A. L. \& Sanson-Fisher, R. W. (1987). Determinants of noncompliance with short-term antibiotic regimens. British Medical Journal 295, 814-8.

Daniel, R. R., and the Azithromycin Paediatric Study Group. (1990). Efficacy and safety of azithromycin in the treatment of pharyngitis and otitis media in children. In Abstracts of the International Congress on Infectious Diseases, Montreal, 1990. Abstract 187.

Edelstein, P. H. \& Edelstein, M. A. C. (1991). In-vitro activity of azithromycin against clinical isolates of Legionella species. Antimicrobial Agents and Chemotherapy 35, 180-1. 
Felstead, S. J., Daniel, R. \& European Azithromycin Study Group. (1991). Short-course treatment of sinusitis and other upper respiratory tract infections with azithromycin: a comparison with erythromycin and amoxycillin. Journal of International Medical Research 19, 363-72.

Finney, J. W., Friman, P. C., Rapoff, M. A. \& Christophersen, E. R. (1985). Improving compliance with antibiotic regimens for otitis media. Randomized clinical trial in a pediatric clinic. American Journal of Disease in Children 139, 89-95.

Foulds, G., Shepard, R. M. \& Johnson, R. B. (1990). The pharmacokinetics of azithromycin in human serum and tissue. Journal of Antimicrobial Chemotherapy 25, Suppl. A., 73-82.

Gordis, L., Markowitz, M. \& Lilienfeld, A. M. (1969). Why patients don't follow medical advice: a study of children on long-term antistreptococcal prophylaxis. Pediatrics 75, 957-68.

Greenberg, R. N. (1984). Overview of patient compliance with medication dosing: a literature review. Clinical Therapeutics 6, 592-9.

Hooton, T. M. (1991). A comparison of azithromycin and penicillin V for the treatment of streptococcal pharyngitis. American Journal of Medicine 91, Suppl. A. 23S-6S.

Lima, J., Nazarian, L., Charney, E. \& Lahti, C. (1976). Compliance with short-term antimicrobial therapy: some techniques that help. Pediatrics 57, 383-6.

Maskell, J. P., Sefton, A. M. \& Williams, J. D. (1990). Comparative in-vitro activity of azithromycin and erythromycin against Gram-positive cocci, Haemophilus influenzae and anaerobes. Journal of Antimicrobial Chemotherapy 25, Suppl. A, 19-24.

Retsema, J. A., Girard, A., Schelkly, W., Manousos, M., Anderson, M., Bright, G. et al. (1987). Spectrum and mode of action of azithromycin (CP-62,993), a new 15-membered-ring macrolide with improved potency against Gram-negative organisms. Antimicrobial Agents and Chemotherapy 31, $1939-47$.

Rylander, M. \& Hallander, H. O. (1988). In-vitro comparison of the activity of doxycycline, tetracycline, erythromycin and a new macrolide, CP-62,993 against Mycoplasma pneumoniae, Mycoplasma hominis and Ureaplasma urealyticum. Scandinavian Journal of Infectious Diseases 53, Suppl.. 12-7.

Washington, J. A. \& Wilson, W. R. (1985). Erythromycin: a microbial and clinical perspective after 30 years of clinical use. Part 2. Mayo Clinical Proceedings 60, 271-8. . 